\title{
Diagnóstico de componentes bioéticos en el ámbito educativo de Ciencias Biológicas y de la Salud ${ }^{*}$
}

\section{Diagnosis of bioethical components in the field of education in biological and health-sciences}

\section{Diagnóstico de componentes de bioética no âmbito de ensino de Ciências Biológicas e da Saúde}

Fecha de recepción: 3 de agosto de 2016

Fecha de evaluación: 14 de noviembre de 2016

Fecha de aceptación: 30 de noviembre de 2016

Disponible en línea: 5 de diciembre de 2016

\author{
David Sebastián Contreras Islas*** \\ Carlos Kelber Lifshitz ${ }^{* * *}$ \\ Enrique Mendieta Márquez* \\ Marco Aurelio Pérez Hernández $z^{* * * *}$ \\ Dor: http://dx.doi.org/10.18359/rlbi.2137
}

Cómo citar:

Contreras Islas, D. S., Kerbel Lifshitz, C., Mendieta Márquez, E. y Pérez Hernández, M. A. (2016). Diagnóstico de componentes bioéticos en el ámbito educativo de Ciencias Biológicas y de la Salud. Revista Latinoamericana de Bioética, 17(1), 14-35. DoI: http://dx.doi.org/10.18359/rlbi.2137

\footnotetext{
* Artículo de investigación.

* Biólogo Experimental y Licenciado en Filosofía. Jefe de materia de Filosofía, Colegio Alemán Alexander von Humboldt, A.C. Correo electrónico: dcontreras@humboldt.edu.mx. ORCID: http://orcid.org/0000-0001-6079-4074. Ciudad de México, México.

*** Maestro en Formación Permanente Carlos Kerbel Lifshitz. Profesor Titular "B" T. C., Universidad Autónoma Metropolitana, Unidad Iztapalapa. División de Ciencias Biológicas y de la Salud. Departamento de Ciencias de la Salud. Correo electrónico: oso@xanum.uam.mx. ORCID: http:// orcid.org/0000-0003-3220-9178. Ciudad de México, México.

**** Maestro en Biología Experimental. Profesor Titular C, Tiempo Completo Universidad Autónoma Metropolitana, Unidad Iztapalapa. División de Ciencias Biológicas y de la Salud Departamento de Ciencias de la Salud. Correo electrónico: enme@xanum.uam.mx. ORCID: http://orcid. org/0000-0003-539-6537. Ciudad de México, México.

${ }_{* * * * *}$ Biólogo, candidato a Doctor en Ciencias (Biología). Profesor-Investigador Titular C, Universidad Autónoma Metropolitana, Unidad Iztapalapa. Departamento de Biología, Área de Ecología. Correo electrónico: maph@xanum.uam.mx. ORCID: http://orcid.org/0000-0003-0372-0110.Ciudad de México, México.
} 
Diagnóstico de componentes bioéticos en el ámbito educativo de Ciencias Biológicas y de la Salud

\section{Resumen}

Las múltiples crisis ecológico-sociales de la actualidad necesitan que la bioética global se integre a la educación superior en Ciencias Biológicas y de la Salud. Partiendo de esta premisa y con miras a desarrollar estrategias educativas que fortalezcan la adquisición de actitudes bioéticas entre los alumnos de las licenciaturas de la División de Ciencias Biológicas y de la Salud (DCBS) de la Universidad Autónoma Metropolitana, Iztapalapa (UAM-I), nuestro equipo realizó dos estudios entre 2012 y 2014, con el objetivo de conocer el contenido de bioética en planes y programas de las licenciaturas de la DCBS y las actitudes de los alumnos de licenciatura, para los cuales se emplearon una metodología de análisis de coincidencias con palabras clave y un cuestionario tipo Likert. Los resultados sugieren que la bioética no se ha incluido adecuadamente en el contenido de planes y programas, ni ha jugado un papel importante en la formación de las actitudes de los estudiantes que; sin embargo, no son tan desfavorables al respecto. Con base en lo anterior, los autores sugerimos una serie de estrategias educativas que podrían mejorar dicha situación, así como otro tipo de análisis para profundizar en el conocimiento de esta problemática.

Palabras clave: bioética, ciencias biológicas, ciencias de la salud, educación superior.

\section{Abstract}

The multiple ecological and social crisis of today require the integration of global bioethics into higher education in Biological and Health Sciences. Based on this premise and with the intention of developing educational strategies to strengthen the acquisition of bioethical attitudes among the undergraduate students of the Division of Biological Sciences and Health (DCBS) of the Autonomous Metropolitan University, Iztapalapa (UAM-I), our team conducted two studies between 2012 and 2014, aiming to know the content of bioethics in plans and research programs of the degrees offered at the DCBS and the attitudes of undergraduate students. Therefore, we used a methodology of keyword analysis and a Likert questionnaire. Our results suggest that bioethics has not been adequately included as a content of plans and study programs, nor has it played an important role in shaping the attitudes of students. These attitudes, however, are not so unfavorable. Based on the above, we suggest some educational strategies that could improve the situation, as well as another type of analysis to deepen the knowledge of this problem.

Keywords: bioethics, higher education, biological sciences, health sciences.

\section{Resumo}

As múltiplas crises ecológico-sociais da atualidade precisam que a bioética global integre-se ao ensino superior em Ciências Biológicas e da Saúde. Partindo deste principio e com vista a desenvolver estratégias educacionais que fortaleçam a aquisição de atitudes bioéticas entre os estudantes das licenciaturas da Divisão de Ciências Biológicas e da Saúde (DCBS) da Universidade Autônoma Metropolitana, Iztapalapa (UAM-I), nossa equipe realizou dois estudos entre 2012 e 2014, com o objetivo de conhecer o conteúdo da bioética nos planos e programas das licenciaturas da Divisão de Ciências Biológicas e da Saúde e as atitudes de alunos de licenciaturas, para o os quais foram empregados uma metodologia de análise de coincidências com palavras-chave e um questionário do tipo Likert. Os resultados sugerem que a bioética não tem sido adequadamente incluída no conteúdo dos planos e programas, e nem tem desempenhado um papel importante na formação das atitudes dos alunos que; no entanto, não são tão desvantajosos ao respeito. Com base no exposto, os autores sugeriremos uma série de estratégias educacionais que poderiam melhorar dita situação, bem como outras análises para aprofundar na compreensão deste problema.

Palavras-chave: bioética, ciências biológicas, ciências da saúde, educação superior. 


\section{Introducción}

El rápido desarrollo de la ciencia y la tecnología, ligado a las dinámicas económicas y sociales predominantes desde la Revolución Industrial, ha desencadenado problemáticas sociales y ecológicas a nivel planetario que demandan nuevos planteamientos éticos. Ante este panorama, en su libro Bioethics, Bridge to the Future, Van Rensselear Potter (1971) propone la bioética como un nuevo campo de conocimiento interdisciplinario preocupado por la sobrevivencia de la especie humana, capaz de integrar los saberes de la biología, la ecología y la medicina con nuestra capacidad para generar valores y normas de comportamiento (Potter, 1971). Esta bioética global, como Potter la denomina, resultó desplazada durante varias décadas por una visión restringida casi exclusivamente a los problemas surgidos en la investigación clínica y las prácticas médicas. Sin embargo, como escribe Gómez-Heras (2012), la profundización de las múltiples crisis en los últimos años ha llevado a otros autores a redescubrir la visión potteriana de una bioética capaz de orientar las acciones humanas de manera que faciliten el tránsito hacia modelos sociales más justos, más respetuosos de los sistemas biológicos y, en general, más sustentables. Es el caso, por ejemplo, de la filósofa mexicana Juliana González Valenzuela, quien hace énfasis en la necesidad de ampliar el alcance de la bioética para abarcar el estudio de las relaciones del ser humano con todos los sistemas vivos, lo que implica la necesa- ria relación de la bioética con todas las disciplinas de las llamadas ciencias de la vida (biología, biotecnología, genética, bioquímica, etc.), además de la medicina (González-Valenzuela, 2008). En el mismo sentido, Enrique Leff (2002) hace explícito el vínculo entre los aspectos éticos y la búsqueda de organizaciones sociales sustentables, mientras que el filósofo y poeta español Jorge Riechmann (2009) escribe: "Vivimos en un mundo de las muchas crisis. Pero la más básica es la crisis de nuestra relación con la biósfera: aún no hemos aprendido a habitar esta Tierra" (p. 15).

Paralelamente a este redescubrimiento de la bioética global en los círculos académicos, la Unesco (2006), preocupada por las problemáticas anteriormente descritas, hace un llamado, en el artículo 23 de su Declaración universal sobre bioética y derechos humanos, a fomentar la formación bioética y la difusión de esta en todos los planos, especialmente entre los jóvenes. La visión del organismo internacional no solo trasciende el enfoque médico y clínico, sino que además enfatiza en la importancia de la educación para la transmisión de esta nueva visión ética, como puede reconocerse en su plataforma Teaching and Learning for a Sustainable Future (Unesco, 2010), así como en las propuestas de abordajes pedagógicos y docentes para acercar estas temáticas a los alumnos de todos los niveles (Unesco, 2012). El enfoque potteriano de la bioética global es refrendado más recientemente en la publicación titulada Global Bioethics: 
What for?, con motivo del vigésimo aniversario del Programa de Bioética de la Organización (Unesco, 2015).

Atendiendo a los llamados de la Unesco, en 2012 la Universidad Nacional Autónoma de México (unam) y el Consejo Nacional de Ciencia y Tecnología (CONACYT) firmaron un convenio de colaboración con la Comisión Nacional de Bioética (Conbioética) con el objetivo de "llevar a cabo acciones concertadas que contribuyan al mejoramiento, desarrollo e impulso de la actividad académica, la investigación y la difusión relacionadas con la bioética, así como instaurar mecanismos para impulsar la bioética y la ética en la ciencia, la tecnología y la innovación" (Ruiz de Chávez-Guerrero, 2014 , p. 705). Dicho convenio es un reconocimiento de la importancia que ha cobrado la bioética para las universidades como un elemento que debe incorporarse a la formación académica de los futuros profesionistas, a fin de que puedan cumplir plenamente con sus obligaciones y responsabilidades sociales, como señala Flores Cuevas (2014). En esta misma línea, Cantú Martínez (2015) señala que para México resulta imperativo impulsar la bioética en la educación superior, que apunte hacia la formación integral de profesionistas que, adicionalmente a su capacidad científica y técnica, actúen honestamente, objeten la inequidad y procuren la justicia social. Ante la realidad cada vez más frecuente de proyectos educativos que no consideren fundamental la instrucción y repercusión de las decisiones profesionales sobre el bienestar común y la dinámica social en general, las distorsiones de las relaciones interpersonales y el dominio de modelos utilitaristas subyacente en los planes de estudio, resulta imperativa la revisión de los currícula (reales y ocultos) para identificar los elementos que nos permitan formar no solo profesionales exitosos, sino también ciudadanos comprometidos con el capital social y el desarrollo sustentable (Bermúdez de Caicedo, 2006).

Como hemos señalado en publicaciones anteriores, los autores compartimos este punto de vista y consideramos que una educación en ética, que forme individuos críticos, con actitudes y valores inspirados por una bioética global, tiene una importancia central para potenciar el desarrollo de profesionistas capaces de hacer frente a las múltiples crisis de la actualidad (Contreras-Islas et al., 2016). Para ello, no solo es necesario coordinar esfuerzos a nivel nacional, sino además integrarlos a procesos similares que se vienen desarrollando a nivel regional en Latinoamérica y en otras regiones. En México, varias universidades incluyen programas de bioética en sus planes de estudio de licenciatura y posgrado (además de existir algunos planes de posgrado específicos para la formación o especialización en bioética), pero más del $70 \%$ de los temas relacionados se imparten en las áreas de la medicina, la enfermería, la odontología, la psicología, y las ciencias biomédicas (Cano-Valle y Neri-Vela, 2012).

Ante esta situación, defendemos la necesidad de incorporar una visión de 
bioética global de corte potteriano que resulte pertinente para los profesionistas, tanto de las ciencias de la salud, como de las ciencias biológicas en general. Creemos que este tipo de bioética, que va más allá de la mera ética médica para incorporar en su discurso dimensiones ecológicas, evolutivas y sociales, entre otras, puede aportar elementos valiosos para encarar las múltiples crisis contemporáneas. Para ello, debe articularse con estrategias educativas y políticas públicas que la vuelvan un elemento realmente cotidiano en la vida de las personas, pero muy especialmente para el caso de los profesionistas del área de las ciencias biológicas y de las ciencias de la salud, que están regularmente en contacto con entornos en los que la dimensión bioética de la toma de decisiones no solo es más evidente, sino también inevitable (Contreras-Islas et al., 2016).

Teniendo en cuenta estas premisas, nuestro equipo ha realizado, desde 2012, diversos estudios con el objetivo de diagnosticar la integración de la temática bioética en los planes y programas de estudio de las seis licenciaturas que se ofrecen en la División de Ciencias Biológicas y de la Salud (DCBS) de la Universidad Autónoma Metropolitana unidad Iztapalapa (UAM-I), así como conocer las actitudes de los alumnos y docentes respecto a la temática en cuestión. Partimos del supuesto hipotético de que dichas temáticas, no obstante su relevancia actual, se encuentran marginalmente consideradas, tanto en nuestros planes y programas de estudio, como en el proceso de enseñanza-aprendizaje desarrollado en las actividades docentes. El propósito final de nuestro estudio es proponer alternativas educativas que contribuyan a formar alumnos mejor informados, más comprometidos y capaces de actuar de acuerdo con principios y valores bioéticos. Como menciona León Correa (2008), el reto no solo parte de introducir la perspectiva bioética en la formación de los profesionistas, sino además de cómo enseñarla o transmitirla a través de procesos educativos que formen parte del futuro profesionista durante su desarrollo.

En este trabajo, se presentan los resultados obtenidos en las primeras etapas de diagnóstico, realizadas entre 2012 y 2015, y se esbozan algunas perspectivas de lo que podrían ser etapas ulteriores de esta misma investigación, tanto en lo referente a propuestas educativas, como en lo que concierne a la continuación, ampliación y profundización de los análisis que aquí se discuten.

\section{Construyendo un modelo metodológico para el análisis del contenido bioético en el ámbito de la educación superior}

\section{Primera etapa: análisis de contenido de planes y programas}

Tomando como base la metodología empleada por Paulin (2012) y Mendieta et al. (2009), se revisaron los lineamientos para la conducción ética de la 
investigación, la docencia y la difusión en la División de Ciencias Biológicas y de la Salud y el reglamento interno de la Comisión de Ética de la DCBS. A partir de este documento, se generó una lista de 20 palabras clave representativas del concepto de bioética manejado por dicha comisión. La búsqueda de estas palabras, que se presentan en la tabla 1 , se realizó en el cuerpo de los planes y programas de estudio oficiales de las seis licenciaturas que ofrece la DCBS. Al existir la Comisión de Ética desde 2007. y habiendo sido modificados los planes y programas de estudio de las licenciaturas en 2012, se esperaba encontrar que la visión de la Comisión de Ética hubiera permeado en estos.
Las licenciaturas se clasificaron en tres bloques diferentes, de acuerdo con su enfoque, con lo que se agrupó a las licenciaturas de Biología (B) y Biología Experimental (BE) en el bloque A; Producción Animal (PA) e Hidrobiología (H) en el bloque B; finalmente, Ingeniería de los Alimentos (IA) e Ingeniería Bioquímica Industrial (IвI) en el bloque C. En cada uno de estos bloques, se buscaron cinco palabras clave adicionales, consideradas especialmente adecuadas para la orientación del trabajo dentro de cada uno de ellos. Las palabras clave específicas para cada uno de los grupos de licenciaturas, además de los enfoques particulares considerados para cada grupo, pueden apreciarse igualmente en la tabla 1.

Tabla 1. Listado de palabras clave generales y específicas para el análisis de contenido

\begin{tabular}{|c|c|c|c|}
\hline \multicolumn{4}{|c|}{ Palabras clave generales } \\
\hline \multicolumn{4}{|c|}{$\begin{array}{l}\text { Autoconciencia, bioética, compromiso, conciencia, convivencia, cooperación, dignidad, equidad/ } \\
\text { igualdad, ética, justicia, legislación, ley, libertad, moral, norma, principio, regla, respeto, respon- } \\
\text { sabilidad, tolerancia. }\end{array}$} \\
\hline Licenciaturas & $\begin{array}{cc}\mathrm{b} & \mathrm{be} \\
\end{array}$ & $\begin{array}{cc}\mathrm{h} & \mathrm{pa} \\
\end{array}$ & $\begin{array}{ll}\text { ibi } & \text { ia } \\
\end{array}$ \\
\hline $\begin{array}{c}\text { Grupo de } \\
\text { licenciaturas }\end{array}$ & a & $\mathrm{b}$ & c \\
\hline $\begin{array}{l}\text { Enfoque } \\
\text { particular }\end{array}$ & $\begin{array}{l}\text { Comprensión del funciona- } \\
\text { miento de los sistemas bio- } \\
\text { lógicos para la conservación } \\
\text { de su integridad. }\end{array}$ & $\begin{array}{l}\text { Comprensión y aprove- } \\
\text { chamiento responsable } \\
\text { de los sistemas biológicos } \\
\text { para la producción de } \\
\text { interés humano. }\end{array}$ & $\begin{array}{l}\text { Aplicación de conocimien- } \\
\text { tos para la innovación en } \\
\text { tecnologías productivas de } \\
\text { interés socioeconómico. }\end{array}$ \\
\hline $\begin{array}{l}\text { Palabras clave } \\
\text { específicas }\end{array}$ & $\begin{array}{l}\text { Conservación, sustentabili- } \\
\text { dad/sostenibilidad, cuidado } \\
\text { de la comunidad de la vida, } \\
\text { interés colectivo y derecho } \\
\text { a la salud/entorno natural. }\end{array}$ & $\begin{array}{l}\text { Sustentabilidad/sosteni- } \\
\text { bilidad, responsabilidad } \\
\text { social, valoración, cuida- } \\
\text { do de la comunidad de la } \\
\text { vida y trato digno. }\end{array}$ & $\begin{array}{l}\text { Valoración, interés colecti- } \\
\text { vo, prevención, principio } \\
\text { de precaución y responsa- } \\
\text { bilidad social. }\end{array}$ \\
\hline
\end{tabular}

Lista de 20 palabras clave obtenidas a partir del contenido del Reglamento interno y los Lineamientos para la conducción ética en la investigación de la Comisión de Ética de la dcbs, para emplearse en el análisis de contenido de planes y programas. Se incluyen, además, la clasificación de las licenciaturas en bloques de acuerdo con su enfoque particular y las palabras clave adicionales que se consideraron para la búsqueda de contenido en cada uno de los bloques.

Fuente: elaboración propia. 
Las coincidencias de las listas de palabras clave dentro de los planes y programas de estudio de las licenciaturas se determinaron utilizando la función de búsqueda avanzada de Acrobat Reader, para lo cual se introdujo la lista con las 25 palabras específicas para cada bloque, más otros términos relacionados semántica y etimológicamente con dichas palabras. Cada coincidencia con los términos buscados fue evaluada, además, en su contexto semántico para determinar si su uso aludía o no al campo de la bioética. En el caso de encontrarse una misma palabra en más de una de las secciones del programa o del plan de estudios, se consideró que existía una recurrencia, susceptible de indicar una mejor articulación del contenido en el documento. Los resultados obtenidos se cuantificaron y procesaron con la versión 2010 de Microsoft Excel.

\section{Segunda etapa: análisis de actitudes de los alumnos de licenciatura}

Tomando como base las propuestas de Méndez Hinojosa (2006) y Sulbarán (2009), se elaboró un cuestionario compuesto por 47 ítems, 30 de los cuales formaban parte de una escala tipo Likert para la medición de actitudes, considerando en ella los elementos cognitivos (10 ítems), afectivos (10 ítems) y conductuales (10 ítems) que conforman el concepto de actitud (Castro de Bustamante, 2003; Sánchez Fernández y Mesa Franco, 1998). Para la validación del instrumento se realizó una prueba piloto de este aplicándolo a un total de
40 alumnos, que representaban un $10 \%$ aproximado de la muestra calculada (Méndez Hinojosa y Peña Moreno, 2006; Rojas Tejada et al., 1998).

El cuestionario se aplicó entre las semanas dos y cuatro del trimestre 14-I (entre enero y febrero de 2014) y se realizó un sorteo aleatorio para determinar los grupos que se visitarían. Al término de este periodo, se consiguió que un total de 531 alumnos de licenciatura respondieran el cuestionario, cantidad que representa un $13 \%$ del total de 4076 alumnos inscritos a las licenciaturas de la DCBS durante dicho periodo.

Los datos obtenidos de los 531 cuestionarios se procesaron y analizaron empleando el programa IBM SPSS v. 21. La escala tipo Likert para la medición de actitudes se validó empleando la prueba de T de Student, y se realizó la prueba por duplicado para las respuestas de 50 y 120 cuestionarios elegidos al azar, respectivamente. De acuerdo con lo estipulado por Méndez Hinojosa y Peña Moreno (2006), se consideraron válidos únicamente aquellos ítems que tuvieron un valor de $\mathrm{T}$ mayor al reportado en las tablas estándar en al menos una de las pruebas. Es decir, aquellos ítems en los que su frecuencia de respuesta en la muestra fue mayor de lo que se daría por azar.

La escala compuesta únicamente por los ítems validados mediante la prueba de $\mathrm{T}$ de Student fue sometida a la prueba de Alfa de Cronbach y se obtuvo un valor de 0,708, el cual refleja una adecuada 
correlación entre los componentes de la escala y corrobora su validez (Ander-Egg, 1995; Babbie, 2000; Rojas Tejada et al., 1998). Únicamente esos ítems fueron considerados para el resto del análisis de esta sección.

Adicionalmente, se buscaron correlaciones entre los ítems de la sección cuatro del cuestionario (referentes a datos académicos del encuestado) y los ítems de las primeras tres secciones empleando la prueba de Chi-cuadrado. Para aquellos casos en los que el valor de $\mathrm{P}<0,05$ en la prueba de Chi-cuadrado, los datos fueron transformados a un formato de Microsoft Excel 2010 y procesados para obtener representaciones gráficas que permitieran visualizarlos de forma más clara. La aplicación de los cuestionarios se llevó a cabo durante el trimestre 15-P (a principios de 2015) y se realizó de forma voluntaria. El desarrollo de la metodología descrita, así como la totalidad del presente análisis, no requirió la sanción o consentimiento institucional de acuerdo con la normatividad vigente al respecto.

Finalmente, dada la naturaleza de los datos y la metodología empleada en ambas etapas, se realizó una asociación generalizada no cuantitativa entre los contenidos de bioética de los documentos normativos oficiales de la DCBS, de los planes y programas de sus seis licenciaturas y los resultados de las encuestas sobre las actitudes de los alumnos.
¿Es relevante la perspectiva bioética para el desarrollo de planes y programas de estudio y el desempeño de alumnos y docentes en la División de Ciencias Biológicas y de la Salud?

\section{Primera etapa: análisis de contenido de planes y programas}

En cuanto a los resultados obtenidos a partir de la búsqueda de palabras clave en los planes y programas de estudio, la tabla 2 muestra una relación entre el total de unidades de enseñanza aprendizaje (UEA) existentes dentro del plan de estudio de cada una de las licenciaturas (primera columna), contra la cantidad de UEA en cuyos programas oficiales se encontraron coincidencias con las palabras clave (segunda columna); finalmente (tercera columna), la cantidad de UEA en cuyos programas oficiales hubo una reaparición (recurrencia) de una misma palabra clave en al menos dos secciones distintas del documento (por ejemplo, en los objetivos generales, en el contenido desglosado, en la bibliografía, etc.). Consideramos que el último factor (la recurrencia o reaparición de palabras clave dentro de un mismo programa oficial) es un indicador importante de que la temática está articulada formalmente dentro de los contenidos curriculares, lejos de limitarse a ser un elemento retórico o discursivo. Las columnas cuatro y cinco expresan la misma información que las columnas dos y tres, pero en porcentajes. 
Tabla 2. Relación de UEA y coincidencias con palabras clave en los programas de licenciatura de la DCBS

\begin{tabular}{|c|c|c|c|c|c|}
\hline & $\begin{array}{c}\text { UEA totales } \\
\text { en plan de } \\
\text { estudios }\end{array}$ & $\begin{array}{c}\text { UEA con } \\
\text { coincidencias }\end{array}$ & $\begin{array}{c}\text { UEA con } \\
\text { recurrencias }\end{array}$ & $\begin{array}{c}\text { Porcentaje } \\
\text { de UEA con } \\
\text { coincidencias }\end{array}$ & $\begin{array}{c}\text { Porcentaje } \\
\text { de UEA con } \\
\text { recurrencias }\end{array}$ \\
\hline B & 64 & 24 & 5 & $38 \%$ & $8 \%$ \\
\hline be & 74 & 17 & 2 & $23 \%$ & $3 \%$ \\
\hline h & 69 & 39 & 5 & $57 \%$ & $7 \%$ \\
\hline pa & 58 & 28 & 17 & $48 \%$ & $29 \%$ \\
\hline ia & 58 & 20 & 9 & $34 \%$ & $16 \%$ \\
\hline ibi & 59 & 16 & 4 & $27 \%$ & $7 \%$ \\
\hline Total & 382 & 144 & 42 & $38 \%$ & $11 \%$ \\
\hline
\end{tabular}

* Relación de UEA y coincidencias con palabras clave en los programas de licenciatura de la DCBS: en la primera columna, total de UEA en los planes de estudio de cada licenciatura. En la segunda columna, cantidad de programas oficiales de UEA en los que se encontraron coincidencias con la lista de palabras clave. En la tercera columna, cantidad de programas oficiales de UEA en los que una misma palabra clave se encontró de manera recurrente en distintas secciones del programa. En la cuarta columna, porcentaje de programas de UEA que tienen coincidencias con las palabras clave. En la quinta columna, porcentaje de UEA que presentan coincidencias recurrentes con las palabras clave. Leyenda: B: Licenciatura en Biología; BE: Licenciatura en Biología Experimental; н: Licenciatura en Hidrobiología; PA: Licenciatura en Producción Animal; iA: Ingeniería de los Alimentos; IBi: Ingeniería Bioquímica Industrial.

Fuente: elaboración propia.

En total, un $62,3 \%$ de los programas no presentó ninguna coincidencia con las palabras clave, un $26,7 \%$ de estos evidenció coincidencias de forma aislada con alguna de las palabras clave y solo un $11 \%$ mostró coincidencias recurrentes en más de una de sus secciones, con alguna de las palabras clave. La figura 1 presenta de manera gráfica el porcentaje de UEA que en sus respectivos programas oficiales presentaron coincidencias aisladas de las palabras clave (línea continua) o bien coincidencias recurrentes de estas (línea punteada), dentro de cada una de las licenciaturas.
Los resultados obtenidos del análisis del contenido de los lineamientos y el reglamento interno de la Comisión de Ética de la DCBS sugieren que la visión ética que dicho organismo posee es adecuada en gran medida a la bioética en sentido amplio, por la que abogamos, lo que consideramos un aspecto muy favorable dada la naturaleza de las labores docentes e investigativas que se desarrollan en la división. Sin embargo, el análisis de contenido bioético de los planes y programas de estudio modificados en 2012, tomando como base la lista de palabras clave elaboradas a partir de 
los documentos oficiales de la comisión, indican que esta perspectiva bioética ha permeado poco hacia los programas oficiales de las UEA de las seis licenciaturas, como puede apreciarse en la tabla 2 y en la figura 1. Estos resultados prueban que la bioética continúa manejándose como un elemento discursivo, sin llegar a articularse de forma sólida en los contenidos de los planes de estudio de las licenciaturas de la DCBS.

La tabla 2 evidencia también que el contenido de bioética en los programas oficiales de las licenciaturas es mayor en las del bloque B, orientadas hacia la producción, y menor en las ingenierías, agrupadas dentro del bloque $\mathrm{C}$. En el caso del bloque a, se encuentra una gran diferencia entre el contenido bioético de las uEA del plan de estudio de Biología, siendo este mucho mayor que el de Biología Experimental, cuya cantidad de palabras clave en los planes de estudio es tan baja como en el caso de las ingenierías. La figura 1 visualiza las diferencias de contenido bioético dentro de las distintas licenciaturas. Resulta interesante ver cómo al revisar los porcentajes de programas de estudio con contenido incluido de manera recurrente, es posible encontrar diferencias incluso dentro del bloque $\mathrm{B}$, siendo este porcentaje claramente mayor para Producción Animal que para Hidrobiología, lo que habla de la medida en la que dichos temas se han consolidado como elementos formativos dentro de las licenciaturas.

\section{Segunda etapa: análisis de actitudes de los alumnos de licenciatura}

Por cuestiones de espacio, es imposible desglosar aquí los resultados obtenidos del análisis de los 47 ítems del cuestionario; sin embargo, señalamos a continuación algunos de los datos que, a nuestro parecer, resultan especialmente más relevantes.

De los 531 alumnos encuestados durante el trimestre 14-I, 130 (25\%) estaban inscritos a la Licenciatura en Biología (B); 56 (11\%), a la Licenciatura en Biología Experimental (BE); 100 (19 $\%$ ) pertenecían a la Licenciatura en Hidrobiología (H); 29 (5 \%), a la Licenciatura en Producción Animal (PA); 91 (17\%), a la de Ingeniería Bioquímica Industrial (IBI), y finalmente 124 (23 $\%$ ), a Ingeniería en Alimentos (IA). Uno de los alumnos no contestó a qué licenciatura pertenecía. La composición de la muestra según licenciaturas puede verse representada gráficamente en la figura 2. En lo referente al trimestre de ubicación de los alumnos encuestados, 168 (32\%) se ubicaban entre el primer y tercer trimestre de la carrera; 90 (17 $\%$ ), entre el cuarto y el sexto trimestre; 79 (15\%), entre el séptimo y el noveno; 103 (19\%), entre el décimo y el doceavo; 76 (14 \%) llevaban más de doce trimestres en la Uam y 15 (3\%) no sabían o no contestaron. 
Figura 1. Porcentaje de programas de uea de cada licenciatura con contenido de bioética

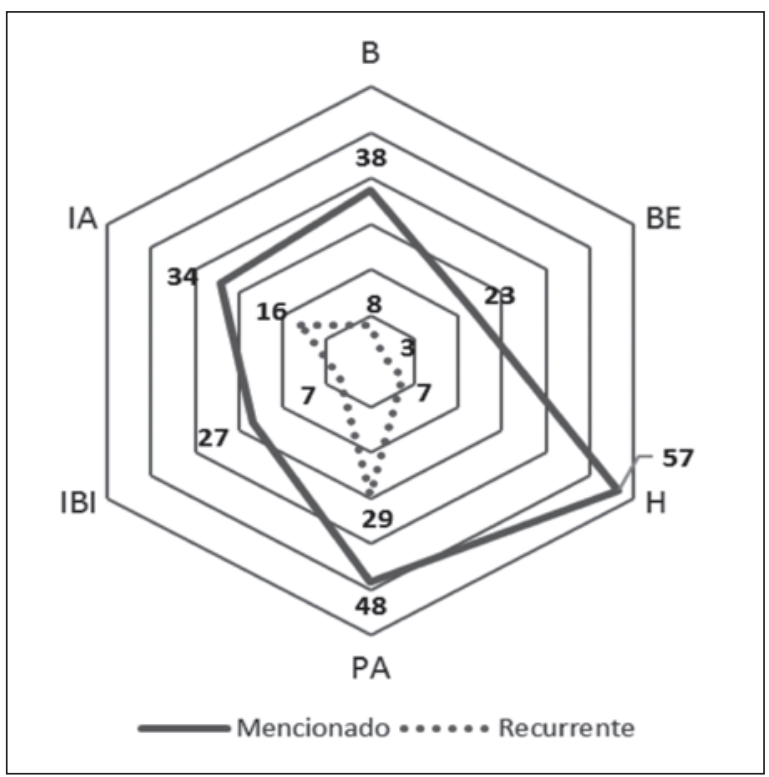

* Porcentaje de programas de uea de cada licenciatura con contenido de bioética: la línea continua se refiere a aquellos programas en los que se encontraron coincidencias aisladas con las palabras clave en alguna de sus secciones. La línea punteada indica el porcentaje de programas en los que se encontraron coincidencias recurrentes en dos o más secciones de estos. Se observa un mayor porcentaje de mención del contenido en las uea de H (57 \%) y pa (48 \%), seguidas por b (38 \%) e ia (34 \%) y finalmente ibi $(27 \%)$ y be $(23 \%)$. En cuanto al contenido recurrente en los programas de uea, los porcentajes más altos los presentan pa (29\%) e ia (16\%), seguidas por b (8\%), h e ibi (7\%) y finalmente be (3\%).

Fuente: elaboración propia.

Un 54 \% del total de la muestra afirmó haber leído, escuchado o utilizado alguna vez el concepto de bioética, mientras que un $46 \%$ lo negó. Sin embargo, solo un $25 \%$ de los alumnos dijo haber recibido información sobre bioética a lo largo de su formación académica y únicamente un $17 \%$ dijo haberla recibido en la UAM. Dichos porcentajes variaron también entre las distintas licenciaturas: así, mientras que un $73 \%$ de los alumnos de в y BE (licenciaturas consideradas como el bloque A dentro del análisis de contenido en planes y programas) dijo haber leído, escuchado o utilizado la palabra bioética, solo un $57 \%$ de los alumnos de н у ра (el bloque в dentro del análisis de contenido en planes y programas) lo constató y, por el contrario, un 59 $\%$ de los alumnos de iBi e iA (el bloque C dentro del análisis de contenido en planes y programas) negó conocer el término. Estas diferencias se muestran en la figura 3. El comportamiento fue 
similar en el caso de los alumnos que dijeron haber recibido o no información a lo largo de su formación académica, y en este punto también hubo diferencias apreciables entre los porcentajes de alumnos que afirmaron haber participado de dicha información a lo largo de su formación académica, especialmente entre los alumnos de las licenciaturas del bloque $\mathrm{A}$ y el bloque $\mathrm{C}$, según la clasificación realizada para el análisis de contenido en planes y programas de estudio. Estos datos pueden apreciarse en la figura 4.

El ítem II.9 del cuestionario preguntaba a los alumnos si tenían conocimiento de que la DCBS contaba con una Comisión de Ética; únicamente el 6,8\% de los alumnos encuestados respondió esta pregunta afirmativamente.

En el caso de la escala para la medición de actitudes, los resultados obtenidos contrastaron drásticamente con los altos porcentajes de alumnos que afirmaron no haber recibido información de bioética en la UAm (17\%), a lo largo de su formación académica ( $25 \%$ ), e incluso con el porcentaje de alumnos ( $46 \%$ ) que desconocía el concepto de bioética al momento de la aplicación de la encuesta. Más del $70 \%$ de los alumnos encuestados presentaron lo que podría denominarse una actitud favorable hacia temáticas y problemáticas bioéticas, con sus respectivas diferencias para cada licenciatura.

Figura 2. Composición de la muestra de acuerdo a las licenciaturas cursadas por los encuestados

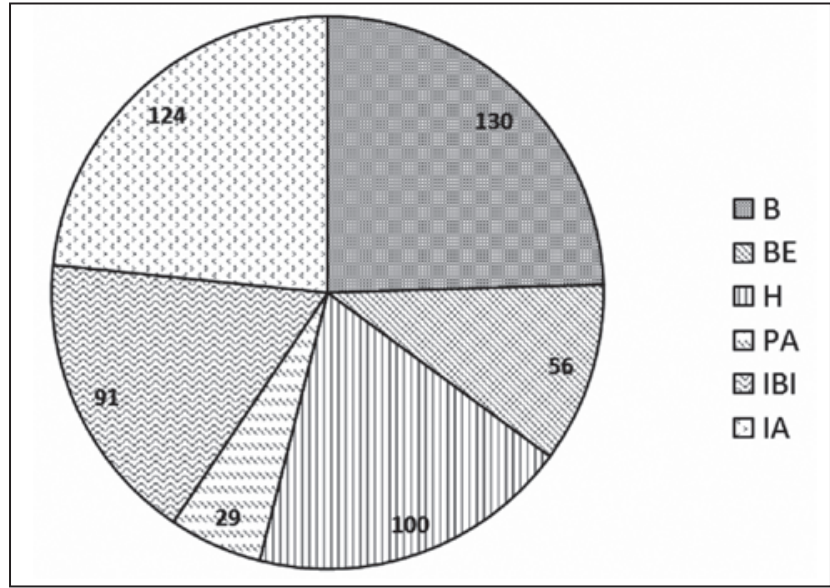

*Composición de la muestra por licenciatura que cursan los encuestados: 130 alumnos (25 \%) pertenecían a Biología (B); 56 (11 \%), a Biología Experimental (BE); 100 (19 \%), a Hirobiología (H); 29 (5\%), a Producción Animal (PA); 91 (17\%), a Ingeniería Bioquímica Industrial (IBI), y 124 (23\%), a Ingeniería en Alimentos (IA). Un alumno no contestó la pregunta (el dato se omitió de la gráfica).

Fuente: elaboración propia. 
Figura 3. Porcentaje de alumnos por licenciatura que dicen haber leído, escuchado o utilizado el término bioética

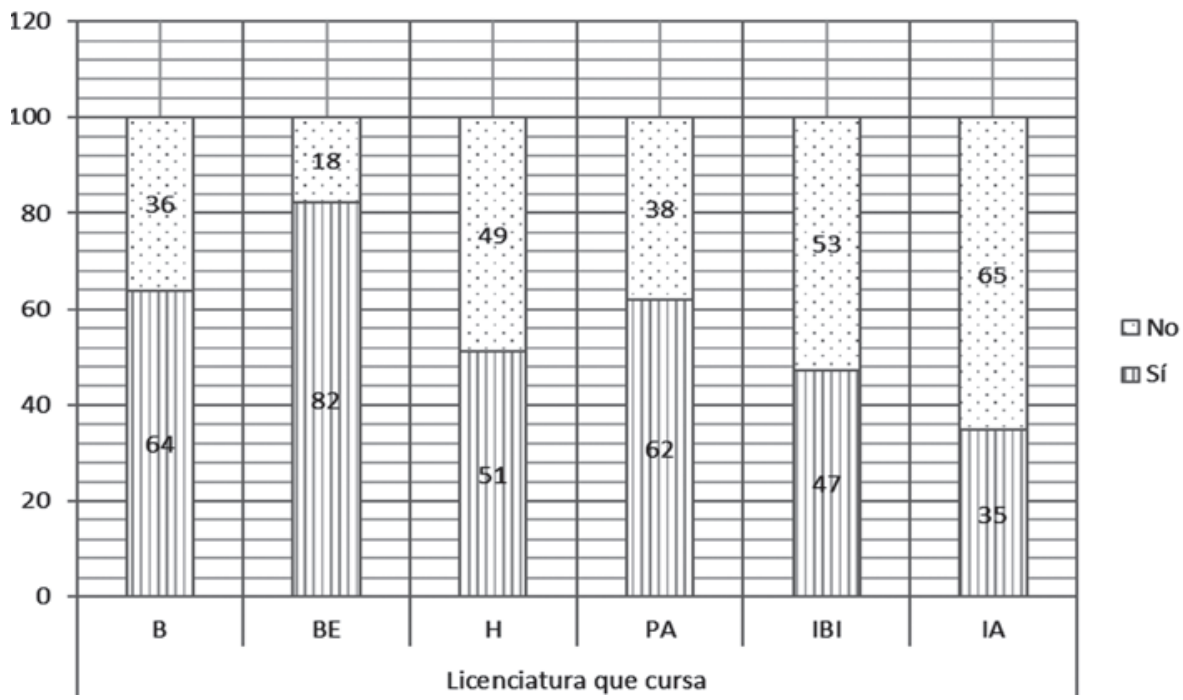

*Porcentaje de alumnos por licenciatura que dicen haber leído, escuchado o utilizado el término bioética: porcentaje de respuestas afirmativas (área con líneas verticales) y negativas (área punteada) recibidas al ítem I.1 del cuestionario: “¿Has escuchado, leído o utilizado el término bioética?”, desglosado para los alumnos encuestados de cada licenciatura. Se aprecia una diferencia importante entre los alumnos de las licenciaturas del bloque A (Biología y Biología Experimental) en comparación con los del bloque C (Ingeniería Bioquímica Industrial e Ingeniería de los Alimentos).

Fuente: elaboración propia.

Así, por ejemplo, un $75 \%$ de los alumnos de licenciaturas clasificadas dentro del bloque A durante el análisis de contenido de planes y programas (Biología y Biología Experimental, respectivamente) presentaron este tipo de actitudes, al igual que el $69 \%$ de los alumnos de las licenciaturas con un enfoque hacia la producción, clasificadas dentro del bloque B (Hidrobiología y Producción Animal), e incluso un 68\% de los alumnos de licenciaturas del bloque C (Ingeniería Bioquímica Industrial e Ingeniería de los Alimentos); estos habían mantenido porcentajes de respuestas negativas más elevados en los otros ítems analizados y contenidos curriculares más pobres, de acuerdo con los resultados de la primera etapa de esta investigación (tabla 2). Una presentación gráfica del porcentaje de los alumnos encuestados de cada licenciatura con actitudes favorables puede encontrarse en la figura $5^{\mathrm{a}}$, y una presentación gráfica del porcentaje de alumnos encuestado de cada licenciatura con actitudes desfavorables se representa en la figura $5 b$. 
Figura 4. Porcentaje de alumnos por licenciatura que afirma haber recibido información sobre bioética como parte de su formación académica

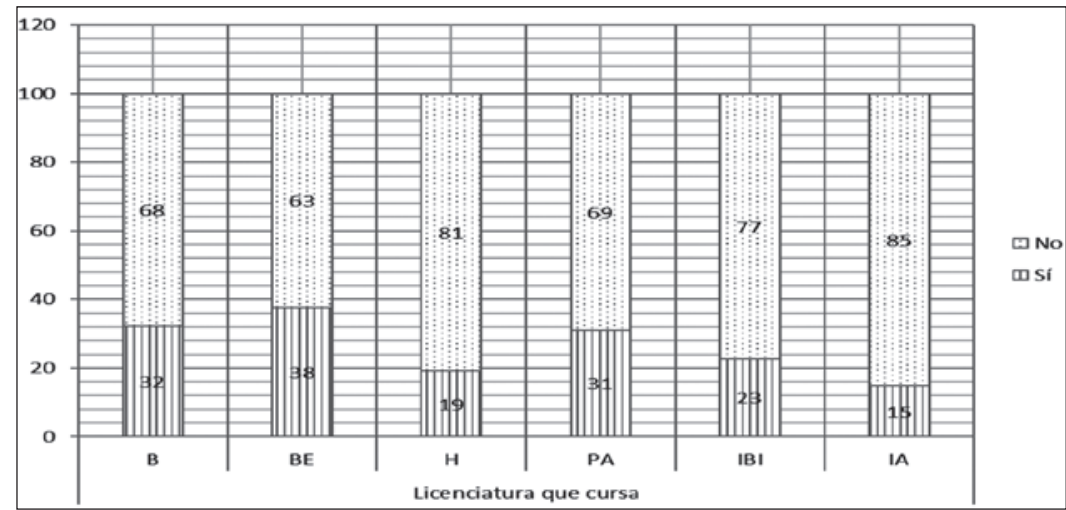

*Porcentaje de alumnos por licenciatura que afirma haber recibido información sobre bioética como parte de su formación académica: porcentaje de respuestas afirmativas (área con líneas verticales) y negativas (área punteada) recibidas a la pregunta II.2 del cuestionario: "¿Has recibido información sobre bioética en tu formación académica?", desglosadas para los alumnos encuestados de cada licenciatura. Se aprecian diferencias importantes entre el porcentaje de respuesta de los alumnos de las licenciaturas del bloque A (Biología y Biología Experimental) en comparación con los del bloque C (Ingeniería Bioquímica Industrial e Ingeniería de los Alimentos).

Fuente: elaboración propia.

Figura 5. Actitudes de los alumnos hacia temáticas de bioética

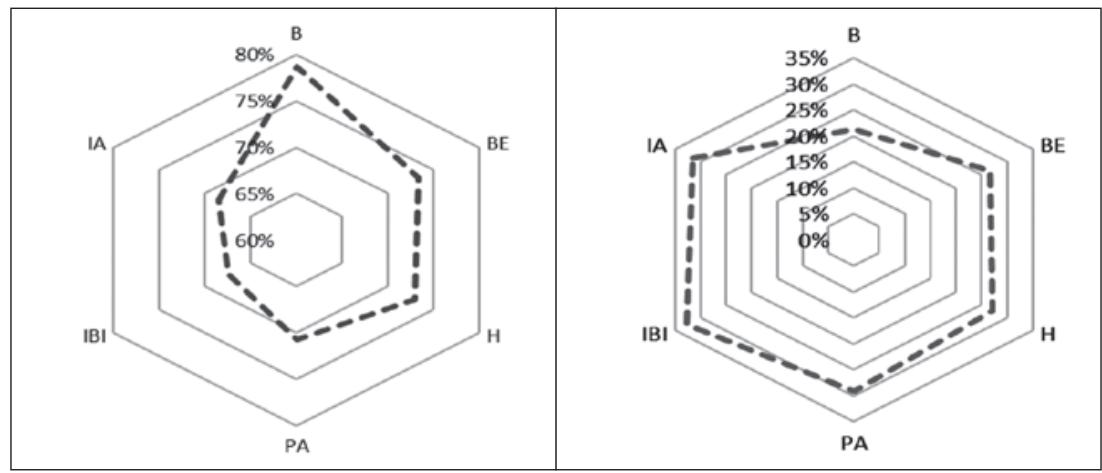

* Del lado izquierdo (a): Porcentaje de alumnos de cada licenciatura que presentan actitudes bioéticas favorables. Porcentaje de actitudes bioéticas favorables de los alumnos encuestados de cada licenciatura. Se presentaron en más del $65 \%$ de los alumnos encuestados, si bien estos porcentajes varían de $79 \%$ para B, a $73 \%$ para BE, $73 \%$ para $\mathrm{H}, 71 \%$ para PA, $67 \%$ para IBI y $69 \%$ para IA. La diferencia entre el porcentaje más elevado de conductas favorables (B) y el más bajo (IBI) fue de 12\%. Del lado derecho (b): Porcentaje de alumnos de cada licenciatura que presentan actitudes bioéticas des favorables. Porcentaje de actitudes bioéticas desfavorables de los alumnos encuestados de cada licenciatura. Se presentan en menos del 35\% de los alumnos encuestados, si bien estos porcentajes varían de $21 \%$ para B, a $27 \%$ para BE, $27 \%$ para $\mathrm{H}, 29 \%$ para $\mathrm{PA}, 33 \%$ para IBI y $31 \%$ para IA. La diferencia entre el porcentaje más bajo de conductas desfavorables (B) y el más alto (IBI) fue de 12\%.

Fuente: elaboración propia. 
De no existir diferencias entre las actitudes de las distintas licenciaturas, la línea discontinua de las figuras $5 \mathrm{a}$ y $5 \mathrm{~b}$ se visualizaría como un hexágono perfecto. Sin embargo, es posible apreciar cómo existe una desviación, en el caso de las actitudes favorables hacia los alumnos de la Licenciatura en Biología (B), cuyo punto se ubica prácticamente sobre el hexágono del $80 \%$, mientras que en el caso de las dos ingenierías (IBI e IA) los puntos del hexágono se ubican por debajo de la línea del 70\%, lo que da como resultado un polígono irregular.

Analizando por separado los componentes cognitivos, afectivos y conductuales que integran el concepto de actitud, fue posible encontrar también diferencias interesantes de los componentes afectivos y cognitivos frente a los conductuales. Los primeros se ubicaron en general entre $12 \mathrm{y}$ 14 puntos porcentuales por encima de los segundos, lo cual sugiere que la mayoría de los alumnos están relativamente bien informados y reaccionan afectivamente de manera favorable hacia cuestiones bioéticas, pero son menos capaces de actuar en conformidad con sus afectos y conocimientos. Esto se ilustra en las figuras 6 a, by c, respectivamente, donde la figura 6a presenta los componentes cognitivos; la figura $6 \mathrm{~b}$, los componentes afectivos, y la figura $6 c$, los componentes conductuales de las actitudes de los alumnos encuestados en cada licenciatura. La irregularidad de los hexágonos de las figuras $6 a$ y $6 \mathrm{~b}$, con relación al de la figura $6 c$, es un reflejo de las diferencias en los componentes mismos.
El contraste de las gráficas de las figuras 5 a y $5 b, 6 a, 6 b, 6 c$ y la figura 1 son especialmente interesantes, pues reflejan una falta de relación entre los contenidos de bioética de los programas de UEA de cada licenciatura y las actitudes de los alumnos de las mismas, en todos sus componentes.

Con las respectivas diferencias encontradas para cada licenciatura, solo un $53,7 \%$ de los alumnos encuestados afirmaron haber leído, escuchado o utilizado el término bioética. De ese porcentaje, aproximadamente la mitad $(24,5 \%)$ aceptó haber recibido dicha información como parte de su formación académica y, nuevamente, únicamente un $70 \%$ de ese $24,5 \%$ ( $17,3 \%$ de la muestra) dijo haber recibido información relativa a la bioética como parte de su formación dentro de la uam. Solo un 6,8 \% del total de la muestra dijo saber de la existencia de la Comisión de Ética de la DCBS y, a pesar de ello, más del $70 \%$ de los alumnos encuestados de cada licenciatura presentaron actitudes favorables a los principios de una bioética potteriana en sentido amplio (Potter, 1971), que es también la perspectiva que la comisión plantea en sus documentos oficiales.

Los resultados aparentemente contradictorios resumidos en el párrafo anterior se prestan para diferentes interpretaciones. Primero que nada, es posible asumir que, aunque la comunidad de alumnos de licenciatura de la DCBS presenta, en efecto, actitudes favorables a la visión de bioética de la propia institución. 
Figura 6. Componentes cognitivos, afectivos y conductuales de las actitudes de los alumnos en relación con la bioética
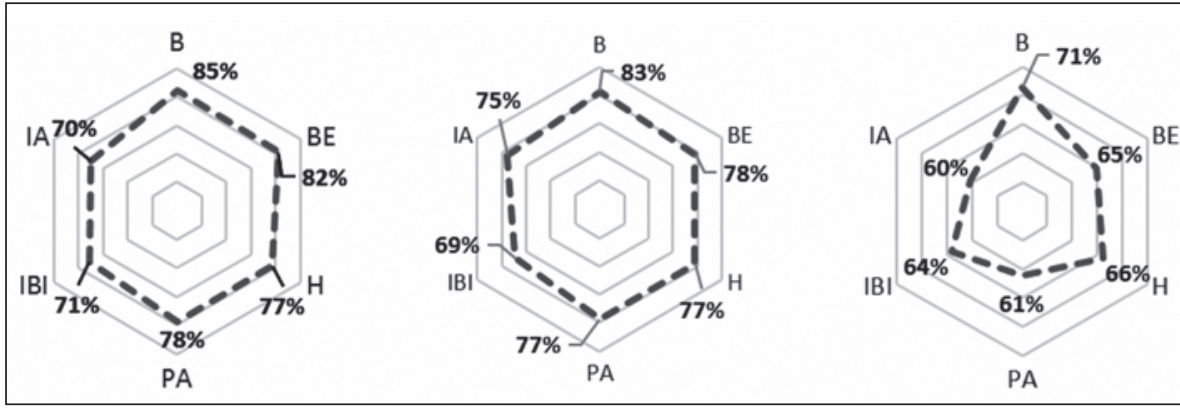

A la izquierda (a): Porcentaje de alumnos encuestados con respuestas favorables en los componentes cognitivos de la escala de medición de actitudes. Al centro (b): Porcentaje de alumnos encuestados con respuestas favorables en los componentes afectivos de la escala de medición de actitudes. A la izquierda (c): Porcentaje de alumnos encuestados con respuestas favorables en los componentes conductuales de la escala de medición de actitudes.

Fuente: elaboración propia.

Esta última ha contribuido muy poco a fomentar dicho aspecto dentro de la formación de sus alumnos, al menos a través de estrategias formales de educación. Esto es tanto más evidente cuando se toman en cuenta los resultados obtenidos del análisis de contenido de los planes y programas de estudio, para lo que el caso de la Licenciatura en Biología Experimental resulta un ejemplo paradigmático, con un contenido bioético recurrente en menos del $25 \%$ de sus programas oficiales, pero un alto porcentaje de alumnos (73\%) con buenas actitudes bioéticas.

Se puede suponer que la buena disposición ante cuestiones bioéticas por parte de los alumnos podría deberse a otros factores presentes en su entorno social, familiar, o bien por su educación preuniversitaria, que contribuyeron a configurar sus actitudes en una dirección favorable hacia la bioética y la sustentabilidad. La situación invita a reflexionar sobre el papel que la propia DCBS, o incluso la Comisión de Ética, podrían o deberían tomar en la difusión de estos temas entre los miembros de la comunidad.

En cuanto a la diferencia encontrada entre los componentes cognitivos, afectivos y conductuales de las actitudes, podemos referirnos a las experiencias de otros autores como François Vallaeys (2009a), quien ha observado la ineficacia que un curso teórico de ética tiene cuando no se cuenta con un espacio en el que sus premisas puedan llevarse a la práctica. El trabajo de Vallaeys, en consonancia con la ética de la virtud propuesta por Aristóteles, parece mostrar que no basta con que los alumnos conozcan lo que es 
"bueno" (de acuerdo, por ejemplo, con una perspectiva bioética como la que sostiene la Comisión de Ética de la DCBS), para que puedan llevarlo a la práctica. Para esto último, es indispensable que el alumno cuente con un entorno donde los principios y valores puedan practicarse y ejercerse. Nuestros resultados, igualmente, sugieren que aunque los alumnos tengan información sobre las cuestiones bioéticas, e incluso respondan de forma afectivamente favorable ante dichas cuestiones, solo llegan a actuar coherentemente con tales conocimientos $y$ afecciones en un porcentaje reducido de los casos. Para incidir sobre esta situación, Vallaeys (2009b) sugiere que el propio campus educativo se configure como un primer espacio donde la ética se aprenda practicándola en la vida diaria.

Cabe preguntarse, ante este panorama, de qué manera podría llevarse a cabo la inserción formal de la cuestión bioética en los planes y programas de estudio, tanto a nivel informativo como formativo; además, de qué estrategia sería adecuada para concederle un nivel de relevancia como el que se les otorga, por ejemplo, a la enseñanza de las matemáticas o de un idioma extranjero (inglés) dentro de los planes de estudio y los programas recientemente revisados y modificados a nivel institucional.

Por otro lado, podemos considerar que, amén de su nivel de conocimiento y actualización en estas temáticas, las actitudes bioéticas son consideradas por los profesores como elementos importantes en su propia formación y desarrollo profesional y que, por tanto, podrían considerar lo mismo para el caso de la formación de los alumnos. Sin embargo, es posible que mucha de esa información, al no ser parte de los planes y programas oficiales, pudiera encontrarse de manera relativamente dispersa y tal vez ser comunicada prácticamente a título personal. Lo anterior lleva a cuestionarse su efectividad, sobre todo al tratar de relacionar este tipo de conocimientos con prácticas que puedan permear a nivel colectivo en toda la comunidad universitaria.

\section{Consideraciones generales y perspectivas}

Los resultados obtenidos hasta ahora indican que, aunque la DCBS a través de su Comisión de Ética cuenta con una perspectiva oficial de bioética bastante favorable, esta ha permeado pobremente hacia los planes y programas de estudio de las licenciaturas, y no se ha integrado de manera consistente a las actividades cotidianas de docentes y a los conocimientos, habilidades y actitudes adquiridas por los alumnos deseablemente aplicables como futuros profesionistas. De allí que consideramos importante que la Comisión de Ética obtenga y ejerza la facultad de revisar y opinar respecto al contenido de los planes y programas de estudio, proponiendo maneras de integrar la temática bioética en ellos de forma más sólida, además de reactivar una de sus atribuciones que es la que le permite organizar talleres, cursos, debates y otras actividades que favorezcan la difusión de información 
sobre el tema. Las actitudes favorables generalizadas de los alumnos que participaron en este estudio sugieren un suelo fértil para recibir y aprovechar este tipo de actividades, las cuáles han sido recientemente iniciadas internamente y han sido recibidas con interés y buena participación de la comunidad estudiantil de la DCBS.

A partir del diagnóstico realizado, uno de los primeros retos que el proyecto tiene por delante es el diseño y la implementación de estrategias y metodologías didácticas complementarias dentro del campo de conocimiento específico de cada licenciatura, que puedan incorporar la cuestión bioética de manera más sólida a la vida académica de los alumnos de la DCBS. Para lograr esto, proponemos comenzar con el diseño y la inserción curricular de un curso obligatorio incluido en el primer año de los planes de estudio, el cual se complementaría más adelante con cursos optativos de bioética con un enfoque más específico para cada una de las orientaciones de las diferentes licenciaturas, durante los últimos años de la carrera. De manera complementaria, pretendemos expandir esta concepción de bioética a otros miembros de la comunidad universitaria, en la forma de algunos cursos de carácter interdisciplinario, utilizando tecnologías de información y comunicación.

Estando conscientes de que el alcance que la educación formal puede tener en la configuración de actitudes (Vallayes, 2009a y 2009b), se ha considerado complementar esta estrategia a través del uso de las redes sociales para abrir otros posibles canales de comunicación, tanto con la comunidad universitaria, como con la población en general. Igualmente, podría considerarse, a futuro, un proyecto de campus sustentable como el que se llevó a cabo en la Uam Xochimilco.

Por lo que al análisis se refiere, es necesario, en primer lugar, concluir con el procesamiento de los datos reunidos a partir de las encuestas realizadas a los profesores durante el trimestre 15-P, esto con el objetivo de saber en qué medida se contaría con el apoyo de este sector de la DCBS para la implementación de estrategias educativas. Igualmente, hemos considerado extender, en el corto plazo, el análisis de actitudes a los directivos y administrativos de la DCBS. A mediano plazo, existe la posibilidad de realizar un estudio similar al que hemos descrito en la DCBS de la Unidad Xochimilco.

Aunque lamentablemente carecemos de estadísticas precisas de seguimiento del desempeño de nuestros egresados, algunas evaluaciones indican que alrededor de un $60 \%$ de ellos entre 1999 y 2002 se desempañaba profesionalmente dentro del rubro o línea de su preparación universitaria. (Rodríguez -Lagunas y Leyva-Piña. 2004). Desde esta perspectiva y para el periodo mencionado, la eficiencia institucional podría parecer razonablemente aceptable, en cuanto a la inserción al mercado laboral e impacto socioeconómico que pudieran generar nuestros profesionistas. No obstante, en el contexto del presente análisis, queda mucho por hacer en cuanto a lo que de su 
desempeño, esos profesionistas, logran integrar al respecto de su responsabilidad bioética y qué tanto de ella debe ser parte de los elementos formativos que adquieren a través de su desempeño académico dentro de nuestras aulas.

Por ello la bioética es un área que interesa al desarrollo en general y la vida universitaria en el ámbito de las ciencias biológicas y de la salud, la cual no está exenta de este proceso formativo debido además a la naturaleza de la relación que cada individuo de nuestra población y especie establece con todas las demás especies del planeta y el ambiente que le rodea, de forma mediata e inmediata y a largo o a corto plazo. La globalización no debe, en estos momentos, entenderse solo definida por vectores económicos o de comunicación, sino que debe ampliarse al espectro ambiental, ecológico y evolutivo, considerando a la especie humana como una más de la diversidad biológica, sujeta, en mayor o menor grado, a los procesos naturales de adaptación y extinción de cualquier otra especie biológica existente.

La educación en ética no es un reto menor; con la bioética, no podría ser diferente. Sin embargo, estamos convencidos de que la inserción de este tipo de temáticas en los contenidos curriculares y, sobre todo, la creación de espacios en los que valores y actitudes bioéticas puedan ponerse en práctica son elementos de gran trascendencia para lograr la transición hacia sociedades más sustentables (Contreras-Islas et al., 2016). Para conseguir la incorporación de estos elementos, sugerimos que las instituciones educativas comiencen con un diagnóstico como el que nuestro equipo ha realizado en la DCBS de la UAM Iztapalapa, pues el conocimiento de las actitudes de alumnos y profesores, así como la visión institucional respecto a la temática (de existir), son factores fundamentales para poder proponer y ejecutar estrategias realmente eficaces.

Aunque nuestro trabajo se ha centrado en el nivel de la educación superior, sería de vital importancia conseguir que la temática bioética y la preocupación por formar seres humanos comprometidos con la sustentabilidad se extendiera hasta los niveles de educación básica, por ser estos algunos de los espacios más importantes en los que hábitos y actitudes toman forma. Desde luego, la educación no formal recibida en casa, e incluso a través de los medios de comunicación, jugaría un papel de igual o incluso mayor relevancia; no obstante, incidir a esos niveles resulta mucho más complejo.

\section{Conclusión}

Aunque la información recopilada y analizada desde el modelo propuesto evidentemente es una primera aproximación, sobre todo en cuanto al análisis y la evaluación curricular, parte de ella, al igual que la derivada de las actitudes de alumnos, nos permite afirmar que el supuesto hipotético planteado en principio no puede ser rechazado. Es decir, que los componentes bioéticos en el 
ámbito educativo de Ciencias Biológicas y de la Salud en nuestras licenciaturas no pueden ser considerados por ahora como elementos integrales de la preparación, formación y futuro desempeño de nuestros egresados.

La propuesta general, a partir de la presente experiencia, es lograr insertar a la bioética en el proceso formativo a nivel de educación superior, como un cúmulo de conocimientos que abarque, en principio, tres elementos: conocimientos interdisciplinarios que integran diferentes disciplinas; regular el proceso educativo, formativo y la actividad profesional propia de los individuos en función del bienestar común, a través de la modificación de actitudes, hábitos, costumbres o comportamientos no compatibles con la sustentabilidad, y propiciar escenarios conducentes a la conservación biológica en general y al mejoramiento de la calidad de la vida humana en particular.

Para el caso concreto de las licenciaturas de la DCBS y la comunidad académica en su conjunto es imperativo, a corto plazo, integrar de manera congruente la normatividad institucional, los contenidos de planes y programas de estudio, la actividad docente y los conocimientos y habilidades adquiridas por los alumnos a lo largo de su proceso de formación.

Si entendemos que la sustentabilidad y la bioética van de la mano, comprenderemos también que la educación en estos temas es una cuestión prioritaria de cara a las crisis que estamos ya en- frentando a escala planetaria, regional y local. A modo de poder responder ante estos retos de forma digna y racional, es indispensable que nosotros mismos y nuestros hijos adoptemos una actitud de profunda consciencia bioética, para reconocernos con mayor humildad y afrontar nuestra interdependencia con el resto de la comunidad de la vida, la delicada posición en la que nos colocan nuestras capacidades tecnológicas y científicas, y finalmente nuestra gran fragilidad, para así poder actuar en consecuencia.

\section{Referencias}

Ander-Egg, E. (1995). Técnicas de investigación social. Buenos Aires: Lunem.

Babbie, E. (2000). Fundamentos de la investigación social. México, D. F: International Thomson.

Bermúdez de Caicedo, C. (2006). Necesidad de la bioética en la educación superior. Acta Bioethica, 12(1), 35-40.

Cano-Valle, F. y Neri-Vela, R.A. (2012). Educación en bioética en México. En S. M. Vidal (Ed.). La Educación en bioética en América Latina y el Caribe: experiencias realizadas y desafíos futuros (pp. 261-278). Montevideo: Programa para América Latina y el Caribe en Bioética y Ética de la Ciencia, Oficina Regional de la Unesco para América Latina y el Caribe.

Cantú-Martínez, P.C. (2015). Bioética y educación superior en México. Acta Bioethica, 21(1), 45-62.

Castro de Bustamante, J.C. (2003). Teoría general de las actitudes. Recuperado el 
29 de agosto de 2016, de http://www. tdx.cat/bitstream/handle/10803/8906/ 02 CapituloParteI.pdf?sequence $=3$

Contreras-Islas, D., Kerbel-Lifshitz, C., Mendieta-Márquez, E. y Pérez Hernández, M.A. (2016). La bioética y nuestra relación con el planeta. Revista de la Academia Mexicana de Ciencia, 67(2), 42-49.

Flores Cuevas, M. 2014. La bioética en la formación del profesional de Bioanálisis en la Facultad de Ciencias de la Salud. Pedagogía Profesional, 12(2). Recuperado el 30 de diciembre de 2016, de http:// www.pedagogiaprofesional.rimed.cu/ Numeros/Vol12\%20no2/maritza.pdf

Gómez-Heras, J. (2012). Bioética y ecología. Los valores de la naturaleza como norma moral. Madrid: Síntesis.

González-Valenzuela, J. (Comp.) (2008). Perspectivas de bioética. México, D. F: Fondo de Cultura Económica, Universidad Nacional Autónoma de México [UNAM].

Leff, E. (Comp.) (2002). Ética, vida, sustentabilidad. México, D. F: Programa de las Naciones Unidas para el Medio Ambiente [PNUMA].

León-Correa, FJ. (2008). Enseñar bioética: cómo transmitir conocimientos, actitudes y valores. Acta Bioethica, 14(1), 11-18.

Méndez Hinojosa, L. M. y Peña Moreno, J. A. (2006). Manual práctico para el diseño de la escala de Likert. México, D. F: Trillas.

Mendieta-Márquez, E., Cortés-Ortiz, M., Kerbel-Lifshitz, C. y González-Casti1lo, O. (9-10 de septiembre de 2009). Diagnóstico del contenido en las temáticas de sustentabilidad en los planes y programas de estudio de las Licencia- turas en la UAm-Iztapalapa. Foro UAM de Sustentabilidad. México, D.F.

Paulin, G. (2012). Humanidades y universidad. La UnAm desde una intertextualidad humanística, México, D. F:: Universidad Nacional Autónoma de México [unam].

Potter, V.R. (1971). Bioethics: Bridge to the Future. Nueva Jersey: Prentice-Hall.

Riechmann, J. (2009). La habitación de Pascal. Ensayos para fundamentar éticas de suficiencia y políticas de autocontención. Madrid: La Catatara.

Rojas Tejada, A., Fernández-Prados, J. S. y Pérez-Meléndez, C. (1998). Investigar mediante encuestas. Fundamentos teóricos y aspectos prácticos. Madrid: Síntesis.

Rodríguez-Lagunas, J. y Leyva-Piña, M.A. (julio-agosto, 2004). Profesionista y trabajo en México: dilemas y posibilidades. En torno a los egresados de la UAm. El Cotidiano, 20(126), 246-259

Ruiz de Chávez-Guerrero, M.H. (2014). Veinte años de bioética en México: desarrollo y perspectivas de la Comisión Nacional de Bioética. Cirugía y Cirujanos, 82, 6, 699-708.

Sánchez Fernández, S.; Mesa Franco, M.C. (1998). Actitudes hacia la tolerancia y la cooperación en ambientes multiculturales. Evaluación e intervención educativa en un contexto concreto: la ciudad de Melilla. Granada: Editorial Universidad de Granada.

Sulbarán, D. (2009). Medición de actitudes. Caracas: Editorial de la Universidad Central de Venezuela.

Unesco. (2006). Declaración Universal sobre Bioética y Derechos Humanos. Paris: United Nations Educational, Scientific and Cultural Organization. 
Unesco. (2010). Teaching and learning for a sustainable future, Recuperado el 29 de agosto de 2016, de http://www.unesco. org/education/tlsf/

Unesco. (2012). Educación para el desarrollo sostenible. Libro de Consulta. Paris: United Nations Educational, Scientific and Cultural Organization.

Unesco. (2015). Global Bioethics: What for? Twentieth anniversary of Unesco's Bioethics Programme. Paris: United Nations
Educational, Scientific and Cultural Organization.

Vallaeys, F. (2009a). El desafío de enseñar ética en la universidad. Recuperado el 29 de agosto de 2016, de http://www. upch.edu.pe/rector/durs/images/Biblio/ MarcoConceptual/EnsenarEticaRS/eldesafiodeensenareticaenlauniversidad.pdf

Vallaeys, F. (2009b). Responsabilidad social universitaria. Manual de primeros pasos. México, D. F: BIS, McGraw-Hill. 